\title{
Surface EMG Signal Acquisition Analysis and Classification for the Operation of a Prosthetic Limb
}

\author{
Kasun Samarawickrama*, Sadun Ranasinghe, Yasoja Wickramasinghe, Wageesha \\ Mallehevidana, Vidarshi Marasinghe and Kanchana Wijesinghe \\ Department of Electrical and Electronic Engineering, General Sir John Kotelawala Defence University, \\ Ratmalana, Sri Lanka. \\ * Corresponding author. Tel.: +94716354866; email: kgswins@gmail.com \\ Manuscript submitted May 12 2017; accepted July 10, 2017. \\ doi: 10.17706/ijbbb.2018.8.1.32-41
}

\begin{abstract}
Biomedical Signal Processing is one of the key areas in Prosthetics. Electromyogram (EMG) signals are used in Prosthetic designing due to good corporation with biomechanics of human body. The aim of this research is to analyze Surface Electromyogram (SEMG) signal parameters related to upper limb speed and flexion angle for one test subject. SEMG signal acquisition was carried out noninvasively for upper limb elbow flexion with minimal ethical issues. Captured Surface EMG signals were amplified by INA128 amplifier IC and filtered by UAF42 filter IC into $0 \mathrm{~Hz}-500 \mathrm{~Hz}$ frequency range. Beaglebone Black digital signal processing unit was interfaced with MATLAB R2015a Simulink platform for processing of SEMG signals. Offline SEMG signal speed classification was done using Fast Fourier Transformation and Wavelet Transformation along with MATLAB R2015a software to classify elbow flexion with respect to speed. Graphical representation of Amplitude variations in each transformation results were able to distinguish the fast elbow flexion and slow elbow flexion. . Flexion angle was approximately calculated by goniometer and data were acquired using Arduino ATMEGA 2560 microcontroller. Applying Curve fitting algorithm to correlate SEMG signals with flexion angle will be the future studies. Ultimate goal will be a generalized algorithm for speed classification.
\end{abstract}

Key words: Elbow flexion, electromyography, flexion angle, speed analysis.

\section{Introduction}

Three decades of war in Sri Lanka caused tragic loss of people. Most of the war heroes and children had undergone limb amputation to save their lives. Not only the war, vehicle accidents, misconduct surgeries and musculoskeletal diseases result amputated persons. Thereafter these people are rejected from society as differently able persons. Currently amputees wear plastic limb models for cover up but they are not functional.

With the emerging technology engineers are able to design and develop prosthetic limbs. Prosthetic limbs are structurally similar to human limbs also capable of doing limb functions. This is a great solution for limb amputees to gain their normal lives.

Limb prosthetics can be divided into 2 main categories such that upper limb prosthetics and lower limb prosthetics. In this paper, we have focused on upper limb prosthesis. Upper limb prosthetics have more complex functions compared to lower limb prosthetics. There is a huge demand for prosthetic limb designing [1]-[4]. Only drawback is the cost. 
In order to formulate prosthetic designs, researchers studied the human limb motions, structural behavior and orientation in different physiological conditions [5]-[7]. They have identified specific signals which are capable of doing muscle contraction and relaxation for limb movements and change in orientation [8]-[10]. They are known as Electromyogram (EMG) signals. The EMG signal's amplitude lies in between 1-10 mV, making it a considerably weak signal. This signal lies in the frequency range from 20-500 $\mathrm{Hz}$ and most dominant in between 50-150 Hz [11], [12]. EMG signals can be acquired in vivo and in vitro by using electrodes and signal acquisition unit [4], [12], [13].

Surface EMG has become reliable and cost effective method for signal acquisition in limbs. Placing electrodes in appropriate location of skin is an essential criterion to acquire optimum EMG signals [13], [14]. Opinion of Neurophysiologist is essential to find exact electrode arrangement on skin to acquire EMG signals.

Although advanced methods are required for detection, processing, analysis and classification of EMG signal. Constructing signal acquisition unit is effective for a research project. In this paper, we have designed low cost data acquisition unit to acquire EMG signals. Apart from that there are many signal processing units already developed in order to follow changes in physiological environment in human body effectively and efficiently. These signal processing units are compatible to Personal Computer (PC) with the interfacing of software [15]-[18].

Currently different types of signal analysis and classification algorithms are formulated for post processing of EMG signals such that filtering, transforming and modeling [4], [19]. Still limb movement analysis of speeds classification and denoising EMG signals are available area for further research.

In this paper, we have shown the promising signs of Fast Fourier Transformation and Wavelet Transformation for the analysis of speed classification.

Interfacing EMG signals with prosthetic design is not an easy task. Therefore, Trajectory planning strategies are required for prosthetics to perform proper tasks as a healthy human limb [20]-[22].

In future, there will be more advanced prosthetic designs along with high end EMG signals post processing algorithms. Thereby prosthetics will be available in commercial market for affordable price.

\section{Methodology}

\subsection{Implementation of EMG Signal Acquisition Unit}

Characteristics of EMG signals have taken into account to implement the design of acquisition unit. Since EMG signals are weak signals, pre-amplification was done using INA128 instrumentation amplifier. This instrumentation amplifier facilitates adjustable gain, high common mode ejection ratio and high input impedance. Gain was set up to 1000 where input microvolts signal was amplified into millivolts signal. UAF42 universal filter was used for extract frequency range of EMG signals. Low pass filter from $0 \mathrm{~Hz}-500 \mathrm{~Hz}$ was implemented to achieve the task. Two 9V DC batteries were used to construct the dual power supply and power up both these integrated circuits. BNC jacks were used as power line connectors. Capacitors were used for stabilize the power up voltage. PCB design was done after verifying this construction on breadboard.

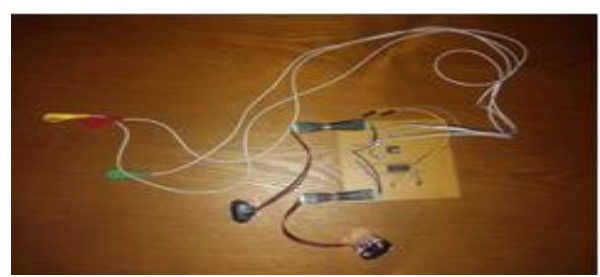

Fig. 1. Complete EMG signal acquisition unit. 
As shown in Fig. 1, the EMG signal acquisition unit implementation was completed. Three inputs from three electrodes were required to capture the EMG signal and output signal pins were designed to connect with the signal processing unit.

\subsection{Selection of Surface EMG Electrodes}

There are 2 methods to acquire EMG signals namely invasive and noninvasive. We have selected noninvasive method due to less ethical issues, minimal infection transmission and patient comfort. We have tested 2 types of noninvasive electrodes to acquire EMG signals. Initially reusable, non adhesive electrodes were used for EMG signals acquisition which is shown in Fig. 2 (a).

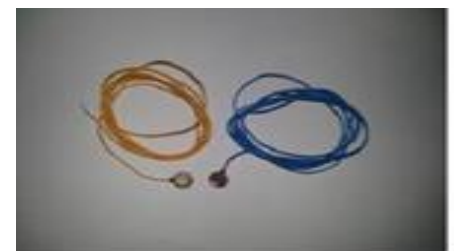

(a)

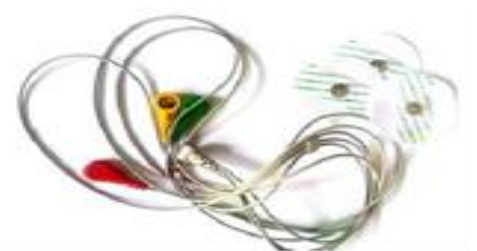

(b)

Fig. 2. Surface EMG electrodes: (a) reusable, non-adhesive, (b) single use, adhesive.

Use of gel was a must to match impedance between skin and electrode. Plasters were used to attach electrode to skin which made patient more uncomfortable. Electrode placement stability wasn't adequate. Then the other type, single use and adhesive electrodes were used for EMG signal acquisition which shown in Fig. 2 (b). These electrodes were gel incorporated and attached to the skin with good stability. Patient comfort was much better compared to earlier stage.

\subsection{Electrode Placement}

In order to capture optimum EMG signal, placing electrodes in appropriate position is critical. Therefore, we have consulted a Neurophysiologist to identify electrode placement of the upper limb. Since our research was focused on to capture EMG signals of elbow flexion, specific electrode placement was shown in Fig. 3.

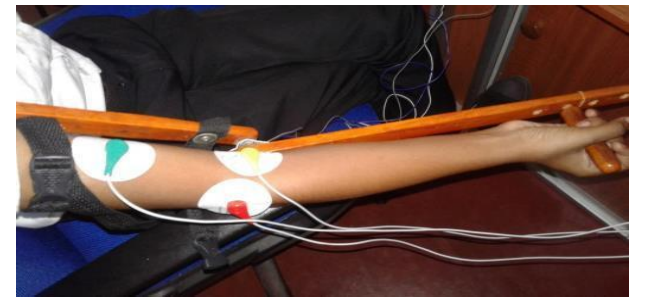

Fig. 3. Electrode placement for elbow flexion.

First electrode (green color) was placed on Bicep Brachaii as EMG signal electrode. Then second electrode (yellow color) was placed on Bicep muscle tendon as reference electrode. Finally, the third electrode (red color) was placed on elbow as ground electrode.

\subsection{Signal Processing Unit}

Beaglebone Black signal processing unit shown in Fig. 4 (a) was selected for this research. Apart from other signal processing units Beaglebone has better advantages. This is a FPGA processing type single board computer which operates from open source hardware. Processing speed can be obtained up to $1000 \mathrm{MHz}$ and low power consuming. Most importantly it is compatible with MATLAB R2015a software which we 
used for EMG signal analysis.

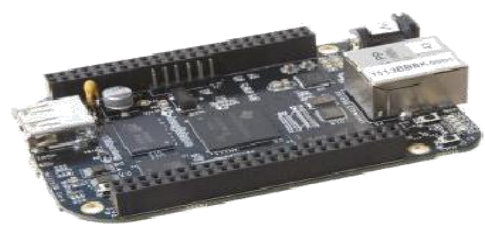

(a)

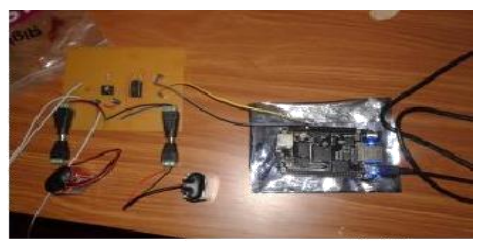

(b)

Fig. 4. Signal processing unit (a) Beaglebone black, (b) Beaglebone black interfaced with EMG signal acquisition unit.

There are 7 analog input ports in Beaglebone where the output pins of EMG signal acquisition unit were connected as shown in Fig. 4 (b). USB cable was connected to PC to power up the signal processing unit as well as to establish the communication between Beaglebone and PC. Analog Input EMG signal was converted into Digital signal inside the Beaglebone and data was outputted via the USB to PC.

\subsection{Signal Visualize and Storage}

In order to visualize the processed EMG signals, MATLAB R2015a software was selected as it is compatible with Beaglebone. To establish the communication between MATLAB and Beaglebone hardware support package had to be installed in MATLAB. Thereby special operating commands were given by MATLAB to establish the communication with Beaglebone. Rather using usual MATLAB command window, we have used MATLAB Simulink library for the signal visualization and storage. In this platform, each command is represented by a block. Thereby we were able to create graphical architecture for visualize EMG signals. Acquired EMG signals were passed through digital filters before plot the output of signal. $50 \mathrm{~Hz}$ and $60 \mathrm{~Hz}$ notch filters were used to eliminate the power line noise. Further $30 \mathrm{~Hz}$ notch filter was used to eliminate baseline noise. Vector scope block was used for visualizing the EMG signal and simout block was used to store the data into MATLAB workspace.

\subsection{Data Collection}

We have selected one voluntary and healthy test subject at age of 24 for this research. In order to analyze the speed classification of elbow flexion 60 data samples were obtained. Among them 30 data samples were slow elbow flexion where the test subject was asked to flex the arm within 6-7 seconds. Rest of the 30 data samples were fast elbow flexion where test subject was asked to flex the arm within 2-3 seconds. Stop watch was provided to test subject to flex the arm within the time period. All the 60 data samples were stored in MATLAB workspace.

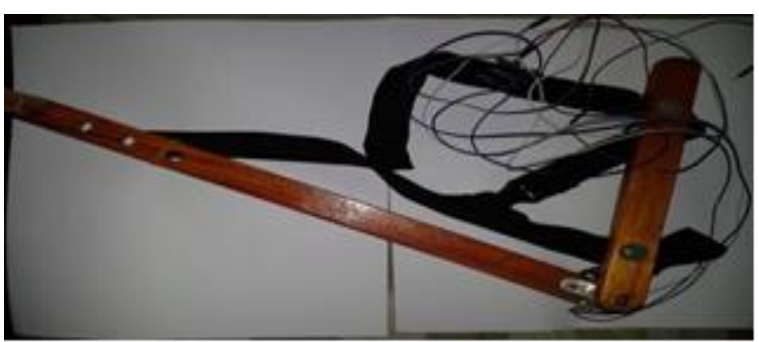

Fig. 5. Goniometer for detection of flexion angle.

\subsection{Detection of Flexion Angle}


In order to perform curve fitting, detection of flexion angle must be recorded parallel to the EMG signal. In this research, Goniometer was designed by using simple potentiometer and two wooden bars with lengths equivalent to test subject's limb measurements. Potentiometer was rotated with the relative movement of wooden bars. Completed design of Goniometer is shown in Fig. 5.

Potentiometer was interfaced to Arduino ATMEGA 2560 and flexion angle data was stored to PC via Arduino.

\subsection{Fourier Transformation}

Discrete or Fast Fourier Transformation was used to analyze time domain EMG signals in frequency domain. Fundamental formula is shown in equation (1).

$$
f(k)=\sum_{n=0}^{N-1} x(n) e^{-j 2 \pi\left(\frac{k n}{N}\right)}
$$

All 60 data samples were transformed into Frequency domain to identify variations between slow elbow flexion and fast elbow flexion.

\subsection{Wavelet Transformation}

Wavelet transformation was used to analyze EMG signal within the time domain. Using Wavelet decomposition, we were able to separate approximation coefficients (low pass components) and detailed coefficients (high pass components). Fundamental formula is shown in equation (2).

$$
\emptyset(x)=\sum_{k=-\infty}^{\infty} a_{k} \emptyset(S x-k)
$$

Since processed EMG signals were discrete signals therefore discrete wavelet transformation was selected for this analysis. Selection of wavelet family was an important point in this research. Daubechies Wavelet family was used thus its wavelet pattern is similar to EMG signal pattern and it can perform discrete wavelet transformation. Average EMG signal of 30 samples in slow elbow flexion and average EMG signal of 30 samples in fast elbow flexion were decomposed by Daubechies Wavelet in order to identify the variations between slow elbow flexion and fast elbow flexion. In this research, we have obtained results up to 2 nd level of decomposition.

\section{Results and Discussion}

\subsection{Verification of EMG signals}

In order to verify signals which obtained from signal acquisition unit as EMG signals, comparison was made with standard EMG signal obtained from standard database.

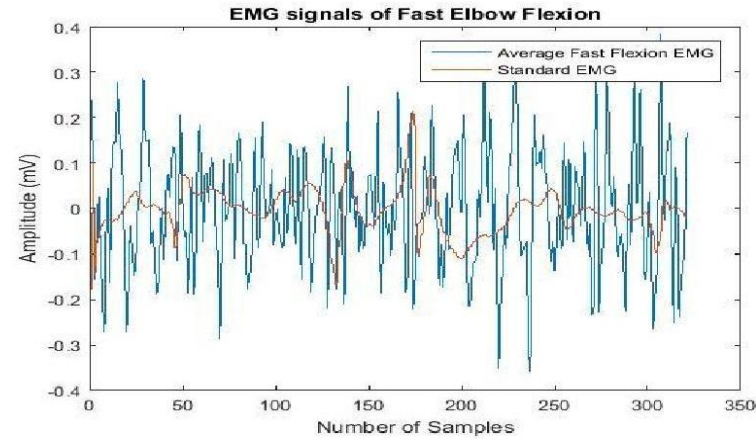

Fig. 6. Fast elbow flexion EMG signal compared with standard EMG signal.

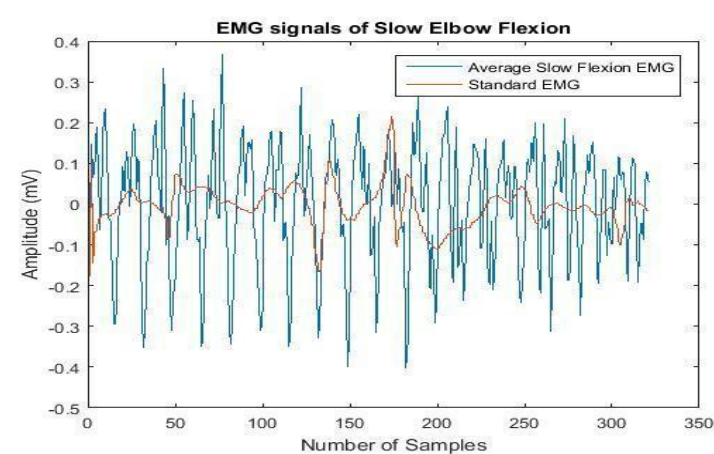

Fig. 7. Slow elbow flexion EMG signal compared with standard EMG signal. 
EMG data of fast elbow flexion and slow elbow flexion were separately compared with standard EMG data as shown in Fig. 6 and Fig. 7. The main observation is both Standard EMG signal and Acquired EMG signals are in millivolts range. Then shape of amplitude fluctuation is quite similar pattern. The reason for acquired EMG signals having higher amplitude value because of mismatch in amplification of EMG signal and adhere of noise. Most importantly acquired signals were verified as EMG signals with above mentioned similarities.

\subsection{Fast Fourier Analysis of EMG Signals}

Collected time domain EMG data samples were converted into frequency domain. Thereby frequency spectrums of each data were obtained as shown in Fig. 8. Slow elbow flexion data were averaged and obtained single frequency spectrum. Same procedure was carried out to fast elbow flexion data.

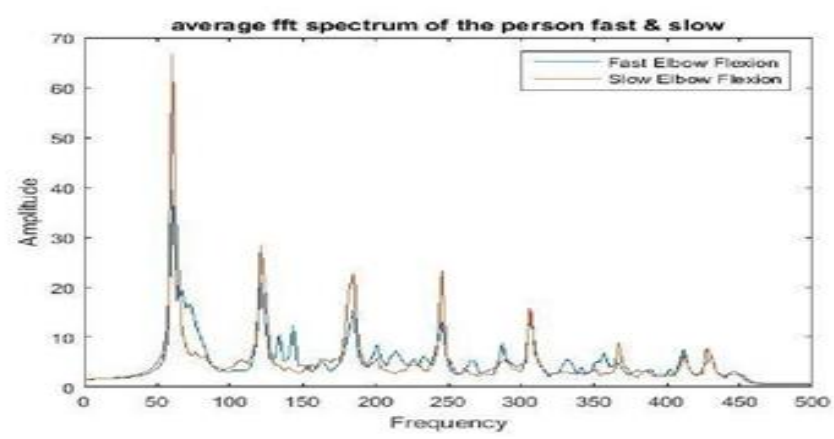

Fig. 8. Averaged frequency spectrums of EMG signals.

Red color frequency spectrum represents slow elbow flexion and Blue color frequency spectrum represents fast elbow flexion. By observing peak values of the frequency spectrum, slow elbow flexion shows higher peak values at low frequencies compared to fast elbow flexion. Thereby we were able to identify the possibility of speed classification using the Fast Fourier Transformation.

\subsection{Wavelet Analysis of EMG Signals}

In wavelet analysis signal domain wasn't changed. This method is more effective than Fast Fourier Transformation due to Analysis is carried out in time domain.

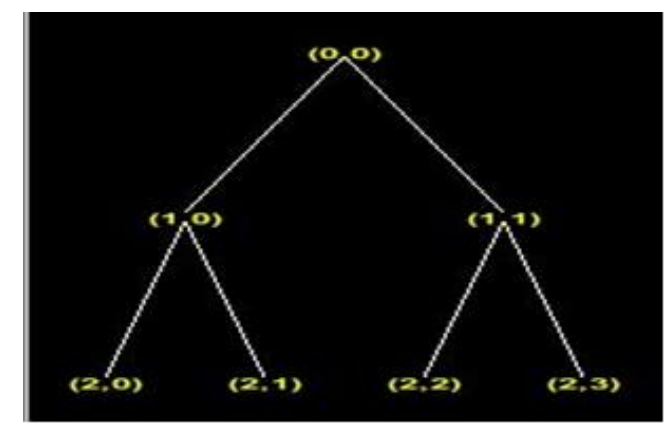

Fig. 9. Wavelet decomposition tree.

Fig. 9 shows Daubechies Wavelet decomposition up to 2nd level. Original signal is represented by $(0,0)$. 1st level of decomposition results are represented by $(1,0)$ and $(1,1)$. 2nd level of decomposition results are represented by $(2,0),(2,1),(2,2)$ and $(2,3)$. In order to obtain results of wavelet decomposition, averaged slow elbow flexion signal and averaged fast elbow flexion signal were obtained. Each signal was decomposed separately and for the comparison identical results were plotted on same graph. Altogether 6 resulted graphs were obtained by Daubechies Wavelet decomposition. 


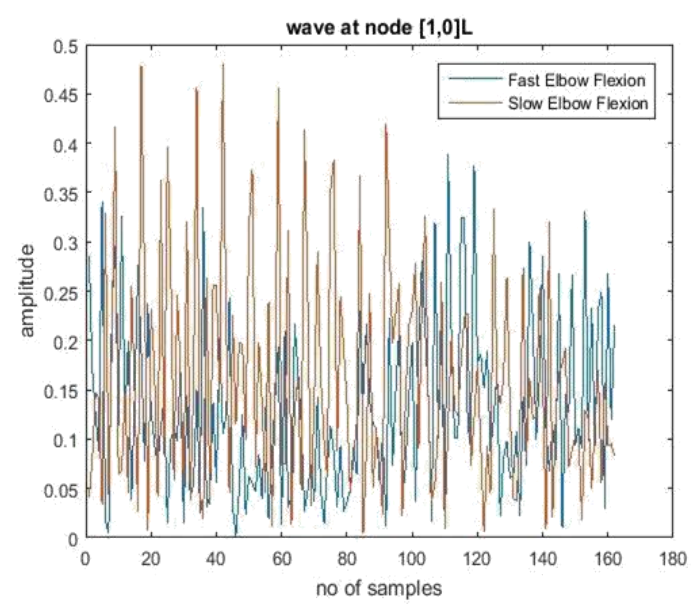

(a). $1^{\text {st }}$ level Approximate coefficients.

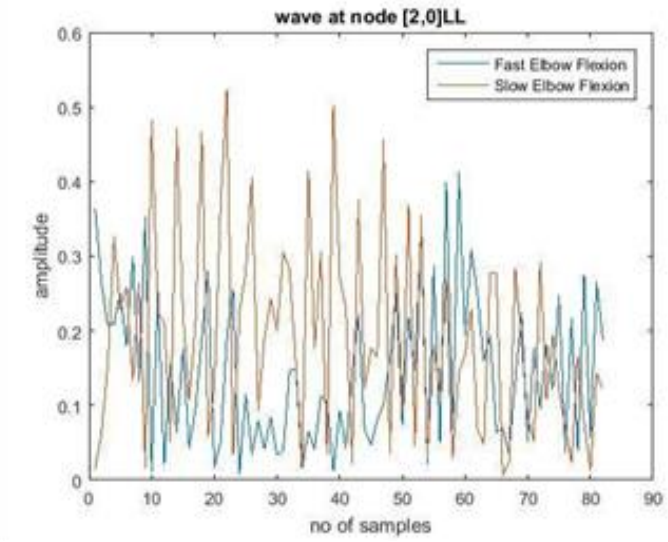

(c). $2^{\text {nd }}$ level Approximate coefficients of 10 (a).

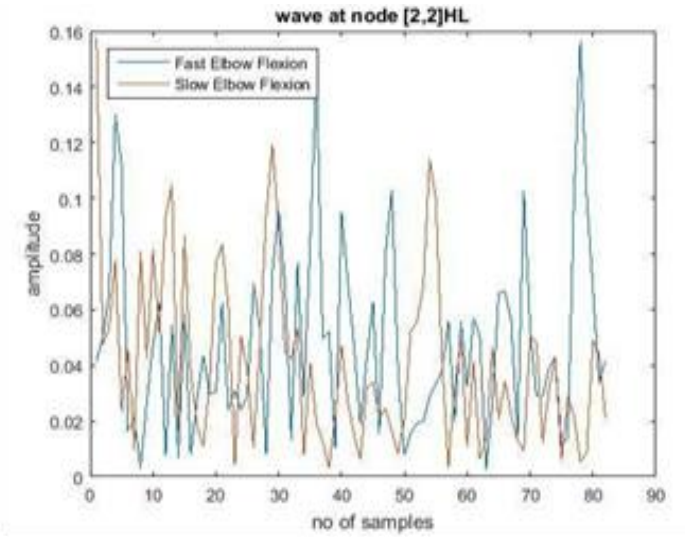

(e). $2^{\text {nd }}$ level Approximate coefficients of 10 (b).

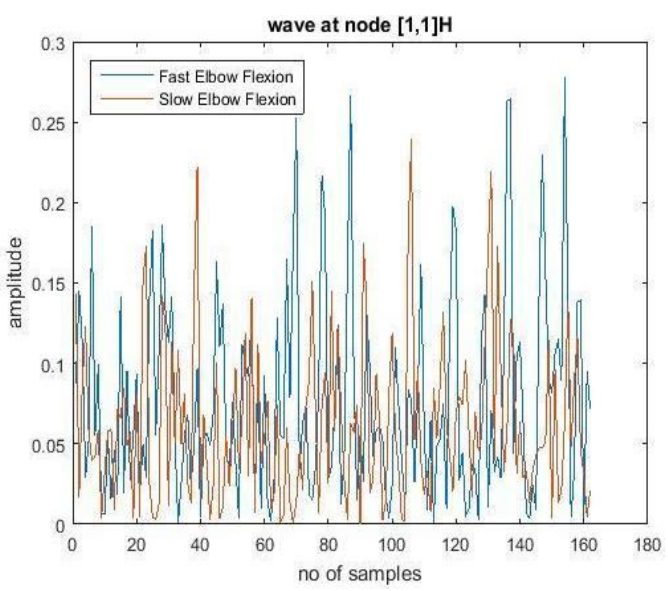

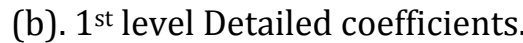

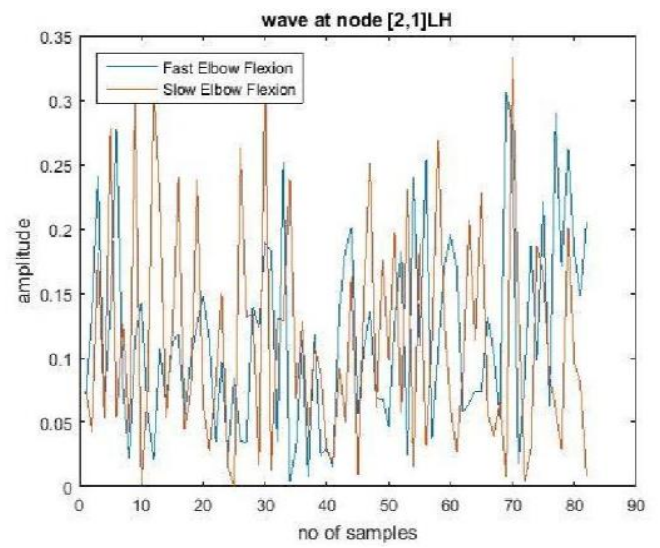

(d). $2^{\text {nd }}$ level Detailed coefficients of 10 (a).

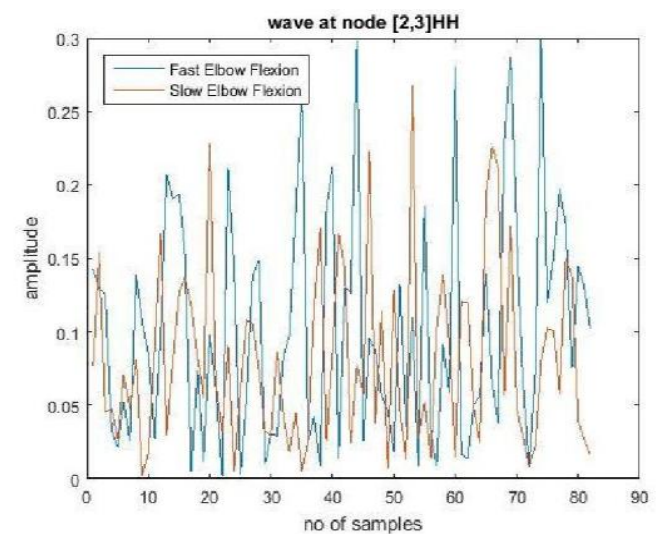

(f). $2^{\text {nd }}$ level Detailed coefficients of 10 (b).

Fig. 10. Wavelet decomposition results.

Slow elbow flexion data were shown in Red color graphs and Fast elbow flexion data were shown in Blue color graphs in Fig. 10. There wasn't any significant difference between red \& blue graphs in Fig. 10 (d) when compared with other graphs. When considering Fig. 10 (a) \& Fig. 10 (c), they give the approximate coefficients values where slow elbow flexion data shows dominant values compared to fast elbow flexion. However Fig.10 (b), Fig. 10 (e) \& Fig. 10 (f) give the detailed coefficients values (Fig. 10 (e) was also derived from 1st level detailed coefficients) where fast elbow flexion shows dominant values compared to slow 
elbow flexion. Thereby we were able to identify the possibility of speed classification using the Wavelet Transformation where low pass component is dominant in slow elbow flexion while high pass components dominant in fast elbow flexion.

4) Angle of flexion with Speed classification

Angle of flexion was recorded by Goniometer for slow elbow flexion and fast elbow flexion parallel to EMG signal acquisition is shown in Fig.11. Extended arm of test subject was considered as the starting point of flexion angle.

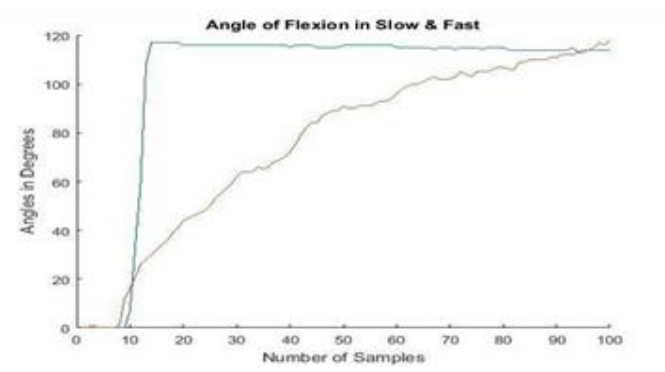

Fig. 11. Recorded flexion angle.

We were able to predict that flexion angle is ranges approximately from $0^{\circ}$ to $120^{\circ}$. During fast elbow flexion test subject was able to reach maximum value within less time (Blue color Graph) compared to slow elbow flexion (Red color Graph). This was the fundamental approach of curve fitting.

\section{Conclusion}

The analysis of speed classification for elbow flexion by surface EMG signals was shown promising results which obtained from Fast Fourier Transformation and Wavelet Transformation. Among these Transformations, Wavelet Transformation analysis is more suitable because EMG signal was able to analyze within time domain. Still Fast Fourier Transformation is effective if anyone required to analyze EMG signals in frequency domain.

Similar analysis can be carried out for elbow extension. Further this analysis can be extended into several test subjects to identify any similarities between EMG signals and Speed classification. The important stage of analysis will be performing this procedure to amputated test subject. Thereby these data can be useful for prosthetic designing for the amputee.

On the other hand, we were able to implement low cost EMG signal acquisition unit. Still it must be improved by optimizing the circuit design and reducing noise interference. Further we suggested using a permanent power supply for future analysis to avoid voltage drop during data acquisition.

Still more development is required for curve fitting and trajectory planning where we suggested combining acquired EMG data with angle of flexion.

\section{Acknowledgment}

The first author thanks goes to staff and students of Biomedical Engineering Intake 30, Department of Electrical, Electronic and Telecommunication Engineering, Faculty of Engineering of General Sir John Kotelawala Defence University, Ratmalana, Sri Lanka providing valuable support and laboratory facilities.

\section{References}

[1] Dellon, B., \& Matsuoka, Y. (2007). Prosthetics, Exoskeletons, and rehabilitation now and for the future.

[2] Gopura, R. A. R. C., Gunasekara, J. M. P. S, T., \& Bandara, D. S. V. (2013) 'Recent trends in EMG-based 
control methods for assistive robots. Electrodiagnosis in New Frontiers of Clinical Research.

[3] Hermens, H., Freriks, B., Disselhorst-Klug, C., \& Rau, G. (2000). Development of recommendations for SEMG sensors and sensor placement procedures. Journal of Electromyography and Kinesiology: Official Journal of the International Society of Electrophysiological Kinesiology, 10(5), 361-74.

[4] Raez, M. B. I., Hussain, M. S., \& Mohd-Yasin, F. (2006). Techniques of EMG signal analysis: Detection, processing, classification and applications. 8 .

[5] Franti, E., Milea, L., Butu, V., Lungu, M., Schiopu, P., Barbilian, A., \& Plavitu, A. (2012). Methods of acquisition and signal processing for myoelectric control of artificial arms. Romanian Journal of Information Science and Technology, 15, 91-105.

[6] Konrad, P. (2010) The ABC of EMG. Retrieved from the website: https://hermanwallace.com/download/The_ABC_of_EMG_by_Pet er_Konrad.pdf

[7] Martin, A. D., Drinkwater, D. T., \& Clarys, J. P. (1984). Human body surface area: Validation of formulae based on a cadaver study. Human Biology, 56(3), 475-488.

[8] Doheny, E., Lowery, M., Fitzpatrick, D., \& O'Malley, M. (2007). Effect of elbow joint angle on force-eMG relationships in human elbow flexor and extensor muscles. Journal of Electromyography and Kinesiology: Official Journal of the International Society of Electrophysiological Kinesiology, 18(5), 760-770.

[9] Edeer, D., \& Martin, C. W. (2011) Upper limb prostheses: A review of the literature: With a focus on myoelectric hands. Richmond, B.C.] : WorkSafeBC, Clinical Services, Worker and Employer Services.

[10] Goen, A., \& Tiwari, D. C. (2015). Classification of the myoelectric signals of movement of forearms for prosthesis control. doi: 10.18178/jomb.5.2.76-84.

[11] Luca, C. J. (2002). Surface electromyography: Detection and recording. Retrieved from the website: https://www.delsys.com/Attachments_pdf/WP_SEMGintro.pdf

[12] Jamal, M. Z. (2012). Computational intelligence in Electromyography analysis - A perspective on current applications and future challenges.

[13] Delsys. (2007). TN101 EMG sensor placement web. Retrieved from the website: http://delsys.com/Attachments_pdf/TN101\%20\%20EMG\%20Sensor\%20Placement-web.pdf

[14] Blanc, Y., \& Dimanico, U. (2010). Electrode placement in surface Electromyography (sEMG) "Minimal Crosstalk Area" (MCA). TOREHJ, 3(1), 110-126.

[15] Instruments, N. (2009) Analyzing your acquired data with LabVIEW. Retrieved from the website: http://www.ni.com/tutorial/9319/en/

[16] MathWorks, T. (1994) MATLAB online - MathWorks United Kingdom. Retrieved from the website: https://in.mathworks.com/products/MATLAB-online/

[17] Roell, J., Sikula, J., \& Desai, J. (2015). Real-time individual finger movement of a Mecha TE robotic hand using human forearm sEMG signals through hardware-software communication. Scholars Journal of Engineering and Technology J. Eng. Tech, 3(3A), 252-257.

[18] Software, S. (2009). The KaleidaGraph Guide to curve fitting. Power, 54.

[19] Alkan, A., \& Günay, M. (2012). Identification of EMG signals using Discriminant analysis and SVM Classifier. 39(1), 44-47.

[20] Bach, H., Dunnigan, M., \& Relay, D. (2008). Position control of a robotic manipulator using a Radial Basis Function Network and a simple vision system. Proceedings of 2008 IEEE International Symposium on Industrial Electronics (pp. 1371-1376).

[21] Gurley. (2009). Numerical methods lecture 5 - Curve fitting techniques. Lecture, Numerical Methods Techniques, 89-102.

[22] Punskaya, E., Andrieu, C., Doucet, A., \& Fitzgerald, W. J. (2002). Bayesian curve fitting using MCMC with 
applications to signal segmentation. IEEE Transactions on Signal Processing, 50(3), 747-758.

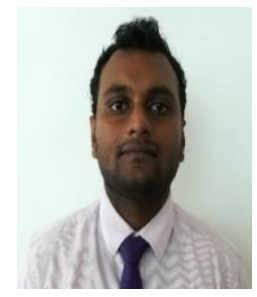

Kasun Samarawickrama was born in Colombo, Sri Lanka in 1993. He completed the BSc. degree in biomedical engineering from General Sir John Kotelawala Defence University, Ratmalana, Colombo, Sri Lanka in 2016.

In 2017, he joined the Department of Electrical, Electronic and Telecommunication Engineering, General Sir John Kotelawala Defence University as a temporary instructor. His current research interests include electromyogram signals, biomedical signal processing and prosthetic designing.

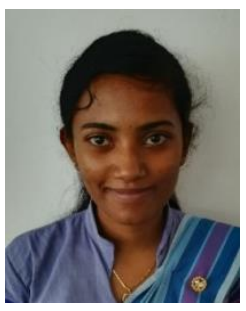

Yasoja Wickramasinghe was born in Badulla, Sri Lanka in 1992. She completed the BSc. degree in biomedical engineering from General Sir John Kotelawala Defence University, Ratmalana, Colombo, Sri Lanka in 2016.

In 2017, she joined the Department of Electrical, Electronic and Telecommunication Engineering, General Sir John Kotelawala Defence University as a temporary instructor. Her current research interests include biomedical signal processing and prosthetic designing.

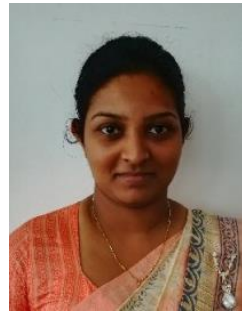

Vidarshi Marasinghe was born in Chilaw, Sri Lanka in 1993. She completed the BSc. degree in biomedical engineering as an officer cadet in Sri Lanka Army from General Sir John Kotelawala Defence University, Ratmalana, Colombo, Sri Lanka in 2016.

In 2017, she joined the Department of Electrical, Electronic and Telecommunication Engineering, General Sir John Kotelawala Defence University as a volunteer instructor. Her current research interests include biomechanics and prosthetic designing.

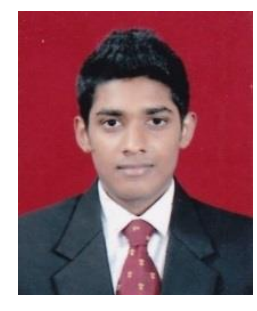

Wageesha Mallehevidana was born in Colombo, Sri Lanka in 1992. He completed the BSc. degree in biomedical engineering from General Sir John Kotelawala Defence University, Ratmalana, Colombo, Sri Lanka in 2016.

In 2017, he joined Sethma Hospitals (pvt) Ltd, Gampaha, Sri Lanka as a biomedical engineer. His current research interests include medical instrumentation and biomechanics.

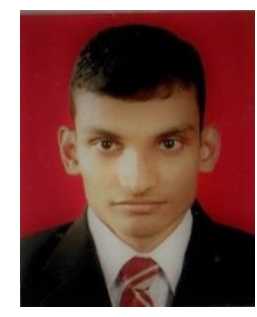

Sadun Ranasinghe was born in Colombo, Sri Lanka in 1992. He completed the BSc. degree in biomedical engineering from General Sir John Kotelawala Defence University, Ratmalana, Colombo, Sri Lanka in 2016.

In 2017, he joined Department of Electronics Engineering, University of Moratuwa, Sri Lanka as a temporary instructor. His current research interests include tissue engineering and prosthetic designing.

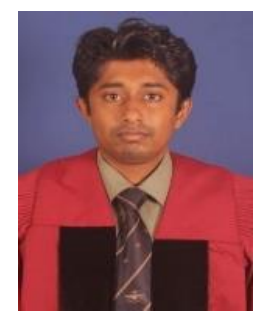

Kanchana Wijesinghe was born in Gampaha, Sri Lanka in 1984. He received the BSc. degree in physical science from University of Colombo, Sri Lanka in 2008, and the MSc. Degree in applied electronics from same University in 2014.

In 2010, he joined Department of Physics, University of Kelaniya, Sri Lanka as an instructor. In 2011, he joined Department of Electrical, Electronics and Telecommunication Engineering, General Sir John Kotelawala Defence University as a lecturer. His current research interests include biomechanics and image processing. 